PROCEEDINGS OF THE

AMERICAN MATHEMATICAL SOCIETY

Volume 133, Number 2, Pages 465-472

S 0002-9939(04)07566-5

Article electronically published on August 30, 2004

\title{
JACOBI POLYNOMIALS FROM COMPATIBILITY CONDITIONS
}

\author{
YANG CHEN AND MOURAD ISMAIL
}

(Communicated by David R. Larson)

\begin{abstract}
We revisit the ladder operators for orthogonal polynomials and re-interpret two supplementary conditions as compatibility conditions of two linear over-determined systems; one involves the variation of the polynomials with respect to the variable $z$ (spectral parameter) and the other a recurrence relation in $n$ (the lattice variable). For the Jacobi weight

$$
w(x)=(1-x)^{\alpha}(1+x)^{\beta}, \quad x \in[-1,1],
$$

we show how to use the compatibility conditions to explicitly determine the recurrence coefficients of the monic Jacobi polynomials.
\end{abstract}

\section{INTRODUCTION AND PRELIMINARIES}

We begin with some notation. Let $P_{n}(x)$ be monic polynomials of degree $n$ in $x$ and orthogonal, with respect to a weight, $w(x), x \in[a, b]$;

$$
\int_{a}^{b} P_{m}(x) P_{n}(x) w(x) d x=h_{n} \delta_{m, n} .
$$

We further assume that $\mathrm{v}^{\prime}(z):=-w^{\prime}(z) / w(z)$ exists and that

$$
y^{n}\left[\mathbf{v}^{\prime}(x)-\mathbf{v}^{\prime}(y)\right] w(y) /(x-y)
$$

is integrable on $[a, b]$ for all $n, n=0,1, \ldots$. From the orthogonality condition there follows the recurrence relation,

$$
z P_{n}(z)=P_{n+1}(z)+\alpha_{n} P_{n}(z)+\beta_{n} P_{n-1}(z), \quad n=0,1, \ldots,
$$

where $\beta_{0} P_{-1}(z):=0, \alpha_{n}, n=0,1,2, \ldots$ is real and $\beta_{n}>0, n=1,2, \ldots$

In this paper we describe a formalism which derives properties of orthogonal polynomials, and their recurrence coefficients, from the knowledge of the weight function. We believe this is a new and interesting approach to orthogonal polynomials. In order to keep this work accessible we will only include the example of Jacobi polynomials. We defer to a future publication, the analysis in the case of the generalized Jacobi weights [8, 11]. In the Jacobi case we find the recurrence relations in $\S 2$. In $\S 3$ we show how our approach leads to the evaluation of monic Jacobi polynomials at $x= \pm 1$. We also show that the evaluation of a Jacobi polynomial at $x=1$ or $x=-1$ leads to explicit representations of the Jacobi polynomials. Closed form expressions for the normalization constants $h_{n}$ are also found.

Received by the editors February 21, 2003 and, in revised form, October 2, 2003.

2000 Mathematics Subject Classification. Primary 33C45; Secondary 42C05.

This research was supported by NSF grant DMS 99-70865 and by EPSRC grant GR/S14108. 
The actions of the ladder operators on $P_{n}(z)$ and $P_{n-1}(z)$ are

$$
\begin{gathered}
\left(\frac{d}{d z}+B_{n}(z)\right) P_{n}(z)=\beta_{n} A_{n}(z) P_{n-1}(z), \\
\left(\frac{d}{d z}-B_{n}(z)-\mathrm{v}^{\prime}(z)\right) P_{n-1}(z)=-A_{n-1}(z) P_{n}(z),
\end{gathered}
$$

with

$$
\begin{aligned}
A_{n}(z):= & \left.\frac{w(y) P_{n}^{2}(y)}{h_{n}(y-z)}\right|_{y=a} ^{y=b}+\frac{1}{h_{n}} \int_{a}^{b} \frac{\mathrm{v}^{\prime}(z)-\mathrm{v}^{\prime}(y)}{z-y} P_{n}^{2}(y) w(y) d y, \\
B_{n}(z):= & \left.\frac{w(y) P_{n}(y) P_{n-1}(y)}{h_{n-1}(y-z)}\right|_{y=a} ^{y=b} \\
& \quad+\frac{1}{h_{n-1}} \int_{a}^{b} \frac{\mathrm{v}^{\prime}(z)-\mathrm{v}^{\prime}(y)}{z-y} P_{n-1}(y) P_{n}(y) w(y) d y,
\end{aligned}
$$

where we have used the supplementary condition,

$$
B_{n+1}(z)+B_{n}(z)=\left(z-\alpha_{n}\right) A_{n}(z)-\mathrm{v}^{\prime}(z),
$$

to arrive at (1.4). The equations (1.3)-(1.6) and the supplementary condition $\left(S_{1}\right)$ were derived by Bonan and Clark [4, Bauldry [3], and Mhaskar [10] for polynomial $\mathrm{v}$, and the authors [7] for general v. Ismail and Wimp [9] identified the additional supplementary condition,

$$
B_{n+1}(z)-B_{n}(z)=\frac{\beta_{n+1} A_{n+1}(z)-\beta_{n} A_{n-1}(z)-1}{z-\alpha_{n}} .
$$

Our thesis in this work is that the supplementary conditions, $\left(S_{1}\right)$ and $\left(S_{2}\right)$, being identities in $n(n>0)$ and $z \in \mathbf{C} \cup\{\infty\}$ have the information needed to determine the recurrence coefficients and other auxiliary quantities. We illustrate this by systematically using $\left(S_{1}\right)$ and $\left(S_{2}\right)$ to determine most of the properties of the Jacobi polynomials. See [14, [2], and [12, for detailed information concerning the Jacobi polynomials. In describing our results we shall follow the standard notation for shifted factorials and hypergeometric functions in [2, 12].

Below, we reinterpret $\left(S_{2}\right)$. We set

$$
\begin{aligned}
\Phi_{n}(z) & :=\left(\begin{array}{c}
P_{n}(z) \\
P_{n-1}(z)
\end{array}\right), \\
\mathrm{M}_{n}(z) & :=\left(\begin{array}{cc}
-B_{n}(z) & \beta_{n} A_{n}(z) \\
-A_{n-1}(z) & B_{n}(z)+\mathrm{v}^{\prime}(z)
\end{array}\right), \\
\mathrm{U}_{n}(z) & :=\left(\begin{array}{cc}
z-\alpha_{n} & -\beta_{n} \\
1 & 0
\end{array}\right) .
\end{aligned}
$$

Now equations (1.3) and (1.4) become,

$$
\Phi_{n}^{\prime}(z)=\mathrm{M}_{n}(z) \Phi_{n}(z),
$$

and the recurrence relations become

$$
\Phi_{n+1}(z)=\mathrm{U}_{n}(z) \Phi_{n}(z) .
$$

We find, by requiring (1.10) and (1.11) to be compatible,

$$
\begin{aligned}
\Phi_{n+1}^{\prime}(z) & =\mathrm{M}_{n+1}(z) \Phi_{n+1}(z) \\
& =\mathrm{M}_{n+1}(z) \mathrm{U}_{n}(z) \Phi_{n}(z) .
\end{aligned}
$$


On the other hand,

$$
\begin{aligned}
\Phi_{n+1}^{\prime}(z) & =\mathrm{U}_{n}^{\prime}(z) \Phi_{n}(z)+\mathrm{U}_{n}(z) \Phi_{n}^{\prime}(z) \\
& =\mathrm{U}_{n}^{\prime}(z) \Phi_{n}(z)+\mathrm{U}_{n}(z) \mathrm{M}_{n}(z) \Phi_{n}(z) .
\end{aligned}
$$

We now write the above equations in matrix form as

$$
\mathrm{S}_{n}(z) \Phi_{n}(z)=0
$$

where $\mathrm{S}_{n}(z)$ is the matrix

$$
\mathrm{S}_{n}(z):=\mathrm{U}_{n}^{\prime}(z)+\mathrm{U}_{n}(z) \mathrm{M}_{n}(z)-\mathrm{M}_{n+1}(z) \mathrm{U}_{n}(z),
$$

whose entries $S_{i, j}$ are

$$
\begin{gathered}
\mathrm{S}_{n}^{11}(z)=1+\left(z-\alpha_{n}\right)\left(B_{n+1}(z)-B_{n}(z)\right) \\
+\beta_{n} A_{n-1}(z)-\beta_{n+1} A_{n+1}(z) \\
\mathrm{S}_{n}^{12}(z)=-\beta_{n}\left(B_{n+1}(z)+B_{n}(z)+\mathrm{v}^{\prime}(z)-\left(z-\alpha_{n}\right) A_{n}(z)\right) \\
\mathrm{S}_{n}^{21}(z)=S_{n}^{12}(z) / \beta_{n} \\
\mathrm{~S}_{n}^{22}(z)=0
\end{gathered}
$$

Here $n=1,2, \ldots$ and $z \in \mathbf{C} \cup\{\infty\}$. Observe that with $\left(S_{1}\right), S_{n}^{12}(z)=S_{n}^{21}(z)=0$. This leaves $S_{n}^{11}(z) P_{n}(z)=0$. Since $P_{n}(z)$ does not vanish identically, we must have $S_{n}^{11}(z)=0$, which is $\left(S_{2}\right)$. It is clear from (1.5) and (1.6) that, if $\mathrm{v}^{\prime}(z)$ is a rational function, then $A_{n}(z)$ and $B_{n}(z)$ are also rational functions. This is particularly useful for our purpose, which is to determine the recurrence coefficients, $\alpha_{n}$ and $\beta_{n}$. In the next section, we illustrate the method by considering the Jacobi weight $w^{(\alpha, \beta)}(x)=(1-x)^{\alpha}(1+x)^{\beta}$ for $x \in[-1,1]$.

Recall that the numerator polynomials [13, [1] are

$$
Q_{n}(z):=\int_{-\infty}^{\infty} \frac{P_{n}(z)-P_{n}(y)}{z-y} w(y) d y
$$

and $\left\{P_{n}(z)\right\}$ and $\left\{Q_{n}(z)\right\}$ form a basis of solutions of the recurrence relation. We shall also use the notation

$$
F(z)=\int_{-\infty}^{\infty} \frac{w(y)}{z-y} d y
$$

for the Stieltjes transform of the weight function.

\section{JACOBI WEIGHT}

The Jacobi weight is $w^{(\alpha, \beta)}(x)=(1-x)^{\alpha}(1+x)^{\beta}, x \in[-1,1]$, and for now we take $\alpha$ and $\beta$ to be strictly positive. It will become clear, using a real analyticity

argument, the results that follow are also valid for $\alpha, \beta>-1$. Let $\left\{\mathcal{P}_{n}^{(\alpha, \beta)}(x)\right\}$ and $\left\{\mathcal{Q}_{n}^{(\alpha, \beta)}(x)\right\}$ denote the monic Jacobi polynomials, and their numerators, respectively; see (1.14). Moreover, in the present example, the Stieltjes transform of $w^{\alpha, \beta}$ will be denoted by $F^{(\alpha, \beta)}(z)$. 
From (1.5)-(1.6) we find

$$
\begin{aligned}
h_{n} A_{n}(z) & =\frac{\alpha}{1-z} \int_{-1}^{1}\left[\mathcal{P}_{n}^{(\alpha \cdot \beta)}(y)\right]^{2}(1-y)^{\alpha-1}(1+y)^{\beta} d y \\
& +\frac{\beta}{1+z} \int_{-1}^{1}\left[\mathcal{P}_{n}^{(\alpha \cdot \beta)}(y)\right]^{2}(1-y)^{\alpha}(1+y)^{\beta-1} d y .
\end{aligned}
$$

Through integration by parts, it readily follows that

$$
A_{n}(z)=-\frac{R_{n}}{z-1}+\frac{R_{n}}{z+1},
$$

for some constant $R_{n}$. Similarly we find

$$
B_{n}(z)=-\frac{n+r_{n}}{z-1}+\frac{r_{n}}{z+1} .
$$

Here $R_{n}$ and $r_{n}$ are given by

$$
\begin{aligned}
R_{n}=R_{n}(\alpha, \beta) & :=\frac{\beta}{h_{n}} \int_{-1}^{1} \frac{\left[\mathcal{P}_{n}^{(\alpha, \beta)}(y)\right]^{2}}{1+y} w^{(\alpha, \beta)}(y) d y, \\
r_{n}=r_{n}(\alpha, \beta) & :=\frac{\beta}{h_{n-1}} \int_{-1}^{1} \frac{\mathcal{P}_{n}^{(\alpha, \beta)}(y) \mathcal{P}_{n-1}^{(\alpha, \beta)}(y)}{1+y} w^{(\alpha, \beta)}(y) d y .
\end{aligned}
$$

It is easy to see that

$$
\begin{aligned}
R_{n}(\alpha, \beta) & =\frac{\beta}{h_{n}} \mathcal{P}_{n}^{(\alpha, \beta)}(-1)\left[\mathcal{Q}_{n}^{(\alpha, \beta)}(-1)-F^{(\alpha, \beta)}(-1) \mathcal{P}_{n}^{(\alpha, \beta)}(-1)\right] \\
r_{n}(\alpha, \beta) & =\frac{\beta}{h_{n-1}} \mathcal{P}_{n-1}^{(\alpha, \beta)}(-1)\left[\mathcal{Q}_{n}^{(\alpha, \beta)}(-1)-F^{(\alpha, \beta)}(-1) \mathcal{P}_{n}^{(\alpha, \beta)}(-1)\right] .
\end{aligned}
$$

The reader may ask, "What is the point of this formalism, since in the attempt to find $\alpha_{n}$ and $\beta_{n}$, two new unknown quantities, $R_{n}$ and $r_{n}$, have been introduced?" However, when $\mathrm{v}^{\prime}$ is a rational function, both sides of $\left(S_{1}\right)$ and $\left(S_{2}\right)$ are rational functions, and by equating coefficients and residues of both sides of $\left(S_{1}\right)$ and $\left(S_{2}\right)$, we shall arrive at four equations, which should be sufficient for the determination of $R_{n}$ and $r_{n}$ as well as $\alpha_{n}$ and $\beta_{n}$. Equating residues of both sides of $\left(S_{1}\right)$, at $z=-1$ and $z=+1$, gives

$$
\begin{aligned}
-2 n-1-r_{n}-r_{n+1} & =\alpha-R_{n}\left(1-\alpha_{n}\right), \\
r_{n}+r_{n+1} & =\beta-R_{n}\left(1+\alpha_{n}\right) .
\end{aligned}
$$

Similarly, from $\left(S_{2}\right)$, we obtain

$$
\begin{aligned}
\left(r_{n}-r_{n+1}-1\right)\left(1-\alpha_{n}\right) & =\beta_{n} R_{-1}-\beta_{n+1} R_{n+1}, \\
\left(r_{n}-r_{n+1}\right)\left(1+\alpha_{n}\right) & =\beta_{n+1} R_{n+1}-\beta_{n} R_{n-1} .
\end{aligned}
$$

Observe that $R_{n}$ can be obtained immediately by adding (2.5) and (2.6):

$$
R_{n}=\frac{1}{2}(\alpha+\beta+2 n+1) .
$$

The sum of (2.7) and (2.8) gives

$$
1-\alpha_{n}=2\left(r_{n}-r_{n+1}\right),
$$

while (2.8) minus (2.7) and with (2.9) gives

$$
\beta-\alpha-2 n-1-(\alpha+\beta+2 n+1) \alpha_{n}=2\left(r_{n}+r_{n+1}\right) .
$$


Now, $(2.10) \pm(2.11)$ implies

$$
\begin{aligned}
4 r_{n} & =\beta-\alpha-2 n-(\alpha+\beta+2 n+2) \alpha_{n}, \\
4 r_{n+1} & =\beta-\alpha-2 n-2-(\alpha+\beta+2 n) \alpha_{n} .
\end{aligned}
$$

When (2.12) and (2.13) are made compatible, we obtain a first-order difference equation satisfied by $\alpha_{n}$ :

$$
\alpha_{n+1}(\alpha+\beta+2 n+4)-\alpha_{n}(\alpha+\beta+2 n)=0,
$$

which has a very simple "integrating factor," $\alpha+\beta+2 n+2$. Using this, we find

$$
\alpha_{n}=\frac{C_{1}}{\left(2 R_{n}-1\right)\left(2 R_{n}+1\right)},
$$

where $C_{1}$ is an "integration" constant, determined by the initial condition

$$
\alpha_{0}=\frac{\mu_{1}}{\mu_{0}}=\frac{\beta-\alpha}{\alpha+\beta+2}, \quad C_{1}=\beta^{2}-\alpha^{2} .
$$

Here $\mu_{j}:=\int_{-1}^{1} t^{j} w(t) d t, j=0,1, \ldots$ are the moments. Therefore we have established

$$
\alpha_{n}=\frac{\beta^{2}-\alpha^{2}}{(\alpha+\beta+2 n)(\alpha+\beta+2 n+2)} .
$$

Going back to (2.8) and using (2.10), we see that $\beta_{n}$ satisfies the linear difference equation:

$$
\beta_{n+1} R_{n+1}-\beta_{n} R_{n-1}=\frac{1-\alpha_{n}^{2}}{2},
$$

which has the "integrating factor" $R_{n}$. Therefore,

$$
\begin{aligned}
\beta_{n} R_{n} R_{n-1} & =C_{2}+\frac{1}{2} \sum_{j=0}^{n-1}\left(1-\alpha_{j}^{2}\right) R_{j} \\
& =C_{2}+\frac{1}{2} \sum_{j=0}^{n-1}\left(1-\frac{C_{1}^{2}}{\left(4 R_{j}^{2}-1\right)^{2}}\right) R_{j},
\end{aligned}
$$

where $C_{2}$ is another integration constant to be determined by the initial condition

$$
\beta_{1}=\frac{h_{1}}{h_{0}}=\frac{h_{1}}{\mu_{0}}=\frac{\mu_{2}}{\mu_{0}}-\left(\frac{\mu_{1}}{\mu_{0}}\right)^{2}=\frac{4(\alpha+1)(\beta+1)}{(\alpha+\beta+2)^{2}(\alpha+\beta+3)} .
$$

After some computations,

$$
C_{2}=\beta_{1} R_{0} R_{1}-\frac{1}{2}\left(1-\alpha_{0}^{2}\right) R_{0}=0 .
$$

Now the sum (2.17) may look complicated; however, with a partial fraction expansion, the sum can be taken and leads to

$$
\beta_{n}=\frac{n(n+\alpha)(n+\beta)(n+\alpha+\beta)}{(2 n+\alpha+\beta)^{2} R_{n} R_{n-1}} .
$$

Therefore, after some simplifications we establish

$$
\beta_{n}=\frac{4 n(n+\alpha)(n+\beta)(n+\alpha+\beta)}{(2 n+\alpha+\beta)^{2}(2 n+\alpha+\beta+1)(2 n+\alpha+\beta-1)} .
$$




\section{EXPLICIT FORMULAS}

We first determine $\mathcal{P}_{n}^{(\alpha, \beta)}( \pm 1)$. Write (1.3) as

$$
\begin{aligned}
\frac{d}{d z} \mathcal{P}_{n}^{(\alpha, \beta)}(z) & =\frac{\left(n+r_{n}\right) \mathcal{P}_{n}^{(\alpha, \beta)}(z)-\beta_{n} R_{n} \mathcal{P}_{n-1}^{(\alpha, \beta)}(z)}{z-1} \\
& +\frac{\beta_{n} R_{n} \mathcal{P}_{n-1}^{(\alpha, \beta)}(z)-r_{n} \mathcal{P}_{n}^{(\alpha, \beta)}(z)}{z+1},
\end{aligned}
$$

and since $\frac{d}{d z} \mathcal{P}_{n}^{(\alpha, \beta)}(z)$ is regular at $z= \pm 1$, we arrive at

$$
\begin{gathered}
\left(n+r_{n}\right) \mathcal{P}_{n}^{(\alpha, \beta)}(1)-\beta_{n} \mathcal{R}_{n} P_{n-1}^{(\alpha, \beta)}(1)=0, \\
\beta_{n} R_{n} \mathcal{P}_{n-1}^{(\alpha, \beta)}(-1)-r_{n} \mathcal{P}_{n}^{(\alpha, \beta)}(-1)=0 .
\end{gathered}
$$

Thus we find

$$
\mathcal{P}_{n}^{(\alpha, \beta)}(1)=\mathcal{P}_{0}^{(\alpha, \beta)}(1) \prod_{j=1}^{n} \frac{\beta_{j} R_{j}}{r_{j}+j}
$$

and

$$
\mathcal{P}_{n}^{(\alpha, \beta)}(-1)=\mathcal{P}_{0}^{(\alpha, \beta)}(-1) \prod_{j=1}^{n} \frac{\beta_{j} R_{j}}{r_{j}} .
$$

Substituting for $\beta_{n}, r_{n}$ and $R_{n}$ from (2.9), (2.12), and (2.18), and applying (2.15) we prove that

$$
\mathcal{P}_{n}^{(\alpha, \beta)}(-1)=\frac{(-1)^{n}}{2^{n}} \prod_{j=1}^{n} \frac{(j+\beta)(j+\alpha+\beta)}{[j+(\alpha+\beta / 2)][j+(\alpha+\beta-1 / 2)]} .
$$

Using the facts $(\lambda)_{n}=\Gamma(\lambda+n) / \Gamma(\lambda),(2 \lambda)_{2 n}=4^{n}(\lambda)_{n}(\lambda+1 / 2)_{n}$ we rewrite the above equation as

$$
\mathcal{P}_{n}^{(\alpha, \beta)}(-1)=\frac{(-1)^{n} 2^{n}(\beta+1)_{n}}{(\alpha+\beta+n+1)_{n}}
$$

Similarly

$$
\mathcal{P}_{n}^{(\alpha, \beta)}(1)=\frac{2^{n}(\alpha+1)_{n}}{(\alpha+\beta+n+1)_{n}} .
$$

We next evaluate $h_{n}$, the squares of the $L^{2}$ norms. In general (1.1) and (1.2) yield $([12])$

$$
h_{n}=h_{0} \beta_{1} \beta_{2} \cdots \beta_{n} .
$$

The beta integral evaluation gives

$$
h_{0}=2^{\alpha+\beta+1} \frac{\Gamma(\alpha+1) \Gamma(\beta+1)}{\Gamma(\alpha+\beta+2)} .
$$

Thus

$$
\int_{-1}^{1} \mathcal{P}_{m}^{(\alpha, \beta)}(x) \mathcal{P}_{n}^{(\alpha, \beta)}(x)(1-x)^{\alpha}(1+x)^{\beta} d x=h_{n} \delta_{m, n}
$$

with

$$
h_{n}=\frac{2^{\alpha+\beta+n+1} \Gamma(\alpha+n+1) \Gamma(\beta+n+1)}{(\alpha+\beta+n+1)_{n} \Gamma(\alpha+\beta+2 n+2)} n ! .
$$


We now prove that

$$
\frac{d}{d z} \mathcal{P}_{n}^{(\alpha, \beta)}(z)=n \mathcal{P}_{n}^{(\alpha+1, \beta+1)}(z) .
$$

For $\alpha>-1, \beta>-1$, and $m<n-1$, integration by parts gives

$$
\begin{gathered}
\int_{-1}^{1} x^{m}\left(\frac{d}{d x} \mathcal{P}_{n}^{(\alpha, \beta)}(x)\right)(1-x)^{\alpha+1}(1+x)^{\beta+1} d x \\
=-\int_{-1}^{1} \mathcal{P}_{n}^{(\alpha, \beta)}(x) f(x)(1-x)^{\alpha}(1+x)^{\beta} d x
\end{gathered}
$$

where $f(x)=x^{m-1}\left[m+x(\beta-\alpha)-x^{2}(\alpha+\beta+m+2)\right]$. Since $f$ has degree at most $n-1$, the above integral must vanish, and we conclude that $\frac{d}{d x} \mathcal{P}_{n}^{(\alpha, \beta)}(x)$ is orthogonal to all polynomials of degree less than $n-1$ with respect to $w^{(\alpha+1, \beta+1)}(x)$. The uniqueness of the orthogonal polynomials and the fact that $\mathcal{P}_{n}^{(\alpha, \beta)}(x), n \geq 0$ are monic establish (3.8). Clearly (3.8) and (3.2) give

$$
\begin{aligned}
\left.\frac{d^{k}}{d x^{k}} \mathcal{P}_{n}^{(\alpha, \beta)}(x)\right|_{x=-1} & =\frac{n !}{(n-k) !} \mathcal{P}_{n-k}^{(k+\alpha, k+\beta)}(-1) \\
& =\frac{n !}{(n-k) !} \frac{(-2)^{n-k}(\beta+k+1)_{n-k}}{(\alpha+\beta+n+k+1)_{n-k}} .
\end{aligned}
$$

The Taylor series about $x=-1$ now gives the representation

$$
\begin{aligned}
& \mathcal{P}_{n}^{(\alpha, \beta)}(x)=\frac{(-2)^{n}(\beta+1)_{n}}{(\alpha+\beta+n+1)_{n}} \\
\times & { }_{2} F_{1}(-n, n+\alpha+\beta+1 ; \beta+1 ;(1+x) / 2),
\end{aligned}
$$

which we recognize to be the monic Jacobi polynomials. Similarly (3.3) and (3.8) give the alternate representation

$$
\begin{aligned}
& \mathcal{P}_{n}^{(\alpha, \beta)}(x)=\frac{(2)^{n}(\alpha+1)_{n}}{(\alpha+\beta+n+1)_{n}} \\
\times & { }_{2} F_{1}(-n, n+\alpha+\beta+1 ; \alpha+1 ;(1-x) / 2) .
\end{aligned}
$$

\section{REFERENCES}

[1] N. I. Akhiezer, The Classical Moment Problem and Some Related Questions in Analysis, English translation, Oliver and Boyed, Edinburgh, 1965. MR 0184042 (32:1518)

[2] G. E. Andrews, R. A. Askey, and R. Roy, Special Functions, Cambridge University Press, Cambridge, 1999. MR 1688958 (2000g:33001)

[3] W. Bauldry, Estimates of asymmetric Freud polynomials on the real line, J. Approximation Theory 63 (1990), 225-237. MR 1079852 (92c:33008)

[4] S. S. Bonan and D. S. Clark, Estimates of the Hermite and the Freud polynomials, J. Approximation Theory 63 (1990), 210-224. MR 1079851 (92c:33007)

[5] S. Bonan, D. S. Lubinsky, and P. Nevai, Derivatives of Orthogonal Polynomials and characterization of weights, in "Approximation Theory 5," Academic Press, New York, 1986, pp. $271-274$.

[6] S. Bonan, D. S. Lubinsky, and P. Nevai, Orthogonal polynomials and their derivatives. II. SIAM J. Math. Anal. 18 (1987), 1163-1176. MR 0892495 (89a:42032)

[7] Y. Chen and M. E. H. Ismail, Ladder operators and differential equations for orthogonal polynomials, J. Phys. A 30 (1997), 7817-7829. MR 1616931 (2000i:33011)

[8] M. E. H. Ismail, An extremal problem for generalized Jacobi polynomials, in "Proc. of the Mathematics Conference, Birzeit University," S. Elaydi et al., eds., World Scientific, 2000, pp. 139-145. MR 1773025 (2001h:33012) 
[9] M. E. H. Ismail and J. Wimp, On differential equations for orthogonal polynomials, Methods and Applications of Analysis 5(4) (1998), 439-452. MR 1669843 (99i:42037)

[10] H. N. Mhaskar, Bounds for Certain Freud-Type Orthogonal Polynomials, J. Approx. Theory 63 (1990), 238-254. MR 1079853 (92c:33009)

[11] P. Nevai, Géza Freud, orthogonal polynomials and Christoffel functions: A case study, J. Approx. Theory 48 (1986), 3-167. MR 0862231 (88b:42032)

[12] E. D. Rainville, Special Functions, Chelsea, the Bronx, 1971. MR 0393590 (52:14399)

[13] J. Shohat and J. D. Tamarkin, The Problem of Moments, revised edition, American Mathematical Society, Providence, 1950. MR 0008438 (5:5c)

[14] G. Szegö, Orthogonal Polynomials, Fourth Edition, Amer. Math. Soc., Providence, 1975. MR 0372517 (51:8724)

Department of Mathematics, Imperial College, 180 Queen's Gate, London SW7 2BZ, UNITED KINGDOM

E-mail address: y.chen@imperial.ac.uk

Department of Mathematics, University of Central Florida, Orlando, Florida 32816

E-mail address: ismail@math.ucf.edu 